JIRSS (2021)

Vol. 20, No. 01, pp 333-345

DOI:10.52547/jirss.20.1.333

\title{
An Alternative to the Beta-Binomial Distribution with Application in Developmental Toxicology
}

\author{
Mehdi Razzaghi ${ }^{1}$ \\ ${ }^{1}$ Department of Mathematics, Bloomsburg University, Bloomsburg, PA, USA.
}

Received: 04/12/2020, Revision received: 07/02/2021, Published online: 03/04/2021

\begin{abstract}
The beta-binomial distribution is resulted when the probability of success per trial in the binomial distribution varies in successive trials and the mixing distribution is from the beta family. For experiments with binary outcomes, often it may happen that observations exhibit some extra binomial variation and occur in clusters. In such experiments the beta-binomial distribution can generally provide an adequate fit to the data. Here, we introduce an alternative when the mixing distribution is assumed to be from the log-Lindley family. The properties of this new model are explored and it is shown that similar to the beta-binomial distribution, the log-Lindley binomial distribution can also be applied in modeling clustered binary outcomes. An example with real experimental data from a developmental toxicity experiment is utilized to provide further illustration.
\end{abstract}

Keywords. Beta-Binomial, Clustered Binary Outcomes, Distribution Mixtures. Extra Binomial Variation, Log-Lindley.

MSC: 62H10, 62H15, 62H20.

Corresponding Author: Mehdi Razzaghi (mrazzagh@bloomu.edu) 


\section{Introduction}

In a sequence of a fixed number $n$ of Bernoulli trials when the success probability $p$ is a random variable, the most common approach is to assume a beta distribution for $p$. In this case the unconditional probability distribution of the number of successes $X$ is the well-known Beta-Binomial model. The flexibility and versatility of the beta distribution in modeling the success probability $p$, makes the beta-binomial family very attractive in modeling. Specifically, when outcomes are dichotomous and observations are in clusters with varying probability of success within each cluster, then the betabinomial model has been successfully applied to model the data in many applications. For example, in ophthalmological studies, the eye is the experimental unit and each individual typically contributes two correlated measurements. Thus, each person is a cluster of size two and this intra-cluster correlation must be accounted for in the analysis of data. One approach is to apply the beta-binomial distribution, see Berson, et al (1980) and Rosner (1982). Similarly, in developmental toxicology experiments with laboratory animals where the effect of a toxin on the offspring of the animal is the outcome of interest, it has been observed that animals from the same litter behave more similar than animals from different litters. Thus, the statistical analysis of data must account for the intra-litter correlation. Williams (1975) was the first person to suggest the application of the beta-binomial model. The properties of the betabinomial distribution are well-known, see for example Wilcox (1981) and Smith (1983). In fact, many modern statistical software namely R (2012) and SAS (2014) have standard functions for fitting the beta-binomial distribution.

Recently, however, there has been some interest in the use of the log-Lindley distribution as an alternative to the beta regression model. The distribution stems from a simple change of variable in the two-parameter extension of the Lindley distribution introduced by Zakerzadeh and Dolati (2010). The log-Lindley distribution was introduced by Gomez-Deniz (2014) and its application in insurance was illustrated. The properties of the distribution were further explored by Jorda and Jimenez-Gamero (2016). Using the log-Lindley distribution, in this paper we derive the log-Lindley binomial mixture and discuss its properties. Similar to the beta-binomial model, we believe that the log-Lindley binomial distribution has many potential applications and can be utilized as an alternative in modeling. Here, we explore the application of the log-Lindley binomial model in developmental toxicology and compare with the results

with the beta-binomial fit. In Section 2 we introduce the log-Lindley distribution and explore its properties in Section 3. In Section 4 we discuss the application in developmental toxicology experiments. Section 5 is devoted to an illustrative example 
based on real experimental data where comparison is made between the applications of the beta-binomial and the log-Lindley binomial models.

\section{The Log-Lindley Binomial Mixture}

The Lindley distribution is a simple one-parameter family of distributions with probability density given by

$$
f(t)=\frac{\alpha^{2}}{\alpha+1}(1+t) e^{-\alpha t}, \quad t \geq 0, \alpha>0
$$

Although the distribution was introduced first in Lindley $(1958,1965)$, it was not until recently that it found widespread popularity. Ghittany et al (2008) discuss several statistical properties of the Lindley distribution and argue that in many ways it is a better model than one based on exponential distribution. The mathematical properties are more flexible and the distribution can prove to be more superior in application. A three-parameter extension of the Lindley distribution was introduced by Zakerzadeh and Dolati (2009) defined by the density

$$
f(t)=\frac{\alpha^{2}(\alpha t)^{\gamma-1}(\theta \gamma+t) e^{-\alpha t}}{(1+\theta \alpha) \Gamma(\gamma+1)}, \quad t \geq 0, \alpha>0, \theta, \gamma \geq 0,
$$

which the authors named the generalized Lindley distribution. Clearly, the addition of the extra two parameters increases the flexibility of the original Lindley model and gives it more versatility and broader application. Note that when $\gamma=\theta=1$ in (2.2), then (2.1) is resulted. Now, if in (2.2) we let $\gamma=1$ and use a change of variable transformation of $U=e^{-T}$, then the density of the random variable $U$ has a log-Lindley distribution and its density is given by

$$
f(u)=\frac{\alpha^{2}}{1+\theta \alpha}(\theta-\ln (u)) u^{\alpha-1}, \quad 0 \leq u \leq 1, \quad \alpha>0, \quad \theta \geq 0 .
$$

Gomez-Deniz et al (2014) discuss several interesting properties of the log-Lindley distribution. In particular they derive closed form expressions for the cumulative distribution function as well as all moments and inverse moments. In addition, they show that by varying the parameters, a wide range of shapes is resulted, making the distribution attractive in modeling as an alternative to the beta family.

Suppose now that the random variable $\mathrm{X}$ has a binomial distribution

$$
p(x \mid \pi)=\left(\begin{array}{l}
x \\
n
\end{array}\right) \pi^{x}(1-\pi)^{n-x}, \quad x=0,1,2, \ldots, n, \quad 0 \leq \pi \leq 1 .
$$


Assume that the success probability $\pi$ is a random variable having a log-Lindley distribution given by (2.3). Then, the unconditional distribution of $X$ is given by

$$
P(x)=\left(\begin{array}{l}
x \\
n
\end{array}\right) \frac{\alpha^{2}}{1+\theta \alpha} \int_{0}^{1} \pi^{x}(1-\pi)^{n-x}(\theta-\ln (\pi)) \pi^{\alpha-1} d \pi,
$$

which after expanding $(1-\pi)^{n-x}$ becomes

$$
P(x)=\left(\begin{array}{l}
x \\
n
\end{array}\right) \frac{\alpha^{2}}{1+\theta \alpha} \sum_{l=0}^{n-x}(-1)^{l}\left(\begin{array}{c}
l \\
n-x
\end{array}\right) \int_{0}^{1}(\theta-\ln (\pi)) \pi^{x+l+\alpha-1} .
$$

After some manipulations, we get

$$
P(x)=\left(\begin{array}{l}
x \\
n
\end{array}\right) \frac{\alpha^{2}}{1+\theta \alpha} \sum_{l=0}^{n-x}(-1)^{l}\left(\begin{array}{c}
l \\
n-x
\end{array}\right) \frac{1+\theta(\alpha+x+l)}{(\alpha+x+l)^{2}} ; \alpha>0, \theta \geq 0, x=0,1,2, \ldots, n,
$$

which is the log-Lindley binomial distribution and can be used as an alternative to the beta-binomial mixture. Although a closed form expression does not exist for the distribution, in practice the computation of the finite sum should not hinder its application. Note that the distribution in (2.5) is somewhat a generalization of what Grassia (1977) called the $G_{1} B D$ distribution. Specifically, when $\theta=0$, the $G_{1} B D$ with $l=2$ is derived. Grassia (1977) suggested the $G_{1} B D$ distribution as an alternative to the beta-binomial model. The inclusion of the additional parameter $\theta$ gives more flexibility to the distribution. Figure 2 displays the shapes of the log-Lindley binomial distribution for various parameter values. In the next section we discuss some of the properties of the distribution given in (2.5).

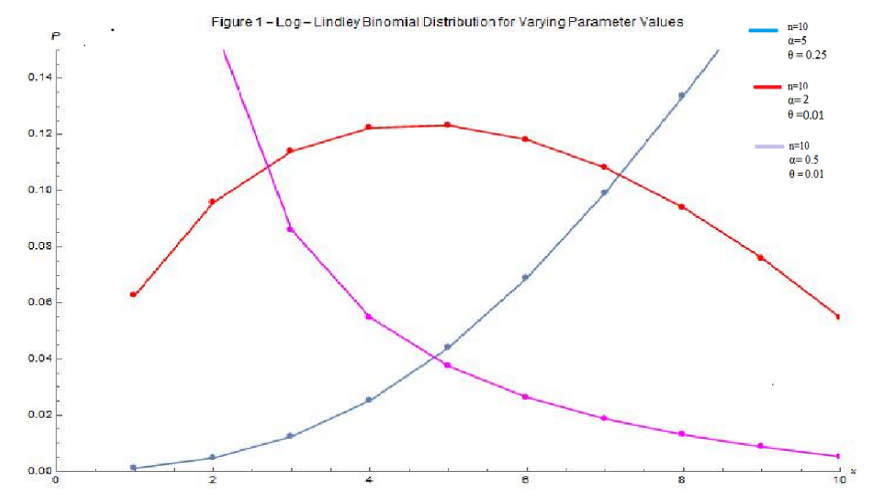

Figure 1: The shapes of the log-Lindley binomial distribution. 


\section{Properties of the Log-Lindley Binomial Distribution}

First, we show that (2.5) indeed defines a valid distribution.

Theorem 3.1. For the log-Lindley binomial distribution given in (2.5),

$$
\sum_{x=0}^{n} P(x)=1
$$

Proof. From (2.5), we have

$$
\begin{aligned}
\sum_{x=0}^{n} P(x) & =\sum_{x=0}^{n}\left(\begin{array}{l}
x \\
n
\end{array}\right) \frac{\alpha^{2}}{1+\theta \alpha} \sum_{l=0}^{n-x}(-1)^{l}\left(\begin{array}{c}
l \\
n-x
\end{array}\right) \frac{1+\theta(\alpha+x+l)}{(\alpha+x+l)^{2}} \\
& =\frac{\alpha^{2}}{1+\theta \alpha}\left\{\sum_{l=0}^{n}(-1)^{l} \frac{1+\theta(\alpha+l)}{(\alpha+l)^{2}}\left(\begin{array}{l}
0 \\
n
\end{array}\right)\left(\begin{array}{l}
l \\
n
\end{array}\right)\right. \\
& \left.+\sum_{l=0}^{n-1}(-1)^{l} \frac{1+\theta(\alpha+x+l)}{(\alpha+x+l)^{2}}\left(\begin{array}{l}
1 \\
n
\end{array}\right)\left(\begin{array}{c}
l \\
n-1
\end{array}\right)+\ldots+\frac{1+\theta(\alpha+n)}{(\alpha+n)^{2}}\right\} .
\end{aligned}
$$

By rearranging, we have

$$
\begin{aligned}
\sum_{x=0}^{n} P(x) & =\sum_{x=0}^{n} \frac{\alpha^{2}}{1+\theta \alpha} \frac{1+\theta(\alpha+x)}{(\alpha+x)^{2}} \sum_{l=0}^{x}(-1)^{j}\left(\begin{array}{l}
l \\
n
\end{array}\right)\left(\begin{array}{l}
x-l \\
n-l
\end{array}\right) \\
& =1+\sum_{x=1}^{n} \frac{\alpha^{2}}{1+\theta \alpha} \frac{1+\theta(\alpha+x)}{(\alpha+x)^{2}}\left(\begin{array}{l}
x \\
n
\end{array}\right) \sum_{l=0}^{x}(-1)^{l}\left(\begin{array}{l}
l \\
x
\end{array}\right)=1 .
\end{aligned}
$$

since

$$
\sum_{l=0}^{x}(-1)^{l}\left(\begin{array}{l}
l \\
x
\end{array}\right)=0
$$

Theorem 3.2. The rth factorial moment of the log-Lindley binomial distribution is given by

$$
\begin{aligned}
h_{r}=E\left[(X)_{r}\right] & =E[X(X-1) \ldots(X-r+1)] \\
& =n(n-1) \ldots(n-r+1)\left(\frac{\alpha}{\alpha+r}\right)^{2} \frac{1+\theta(\alpha+r)}{1+\theta \alpha}, r=1,2, \ldots, n .
\end{aligned}
$$


Proof.

$$
\begin{aligned}
h_{r}= & \sum_{x=0}^{n} X(X-1)(X-2) \ldots(X-r+1)\left(\begin{array}{l}
x \\
n
\end{array}\right) \sum_{l=0}^{n-x}(-1)^{l}\left(\begin{array}{c}
l \\
n-x
\end{array}\right) \frac{\alpha^{2}}{1+\theta \alpha} \frac{1+\theta(\alpha+x+l)}{(\alpha+x+l)^{2}} \\
= & n(n-1)(n-2) \ldots(n-r+1)\left(\frac{\alpha}{\alpha+r}\right)^{2} \frac{1+\theta(\alpha+r)}{1+\theta \alpha} \\
& \times \sum_{x=r}^{n}\left(\begin{array}{l}
x-r \\
n-r
\end{array}\right) \sum_{j=0}^{n-x}(-1)^{l}\left(\begin{array}{c}
l \\
n-x
\end{array}\right) \frac{(\alpha+r)^{2}}{(\alpha+l+x)^{2}} \frac{1+\theta(\alpha+x+l)}{1+\theta(\alpha+r)} \\
= & n(n-1)(n-2) \ldots(n-r+1)\left(\frac{\alpha}{\alpha+r}\right)^{2} \frac{1+\theta(\alpha+r)}{1+\theta \alpha} \\
& \times \sum_{y=0}^{n-r}\left(\begin{array}{c}
y \\
n-r
\end{array}\right) \sum_{l=0}^{n-y-r}(-1)^{l} \frac{(\alpha+r)^{2}}{(\alpha+l+r+y)^{2}} \frac{1+\theta(\alpha+r+l+y)}{1+\theta(\alpha+r)} .
\end{aligned}
$$

By substituting $n^{*}=n-r$ and $\alpha^{*}=\alpha+r$ and using Theorem 3.1, results will follow.

From (3.1), we can easily derive the mean and variance of $X$. By putting $r=1$, we get

$$
\mu_{X}=n\left(\frac{\alpha}{\alpha+1}\right)^{2} \frac{1+\theta(\alpha+1)}{1+\theta \alpha}
$$

and for $r=2$, using $\operatorname{Var}(X)=f_{2}+f_{1}\left(1-f_{1}\right)$, the variance is given by

$$
\sigma_{X}^{2}=n \frac{\alpha^{2}}{1+\theta \alpha}\left\{(n-1) \frac{1+\theta(\alpha+2)}{(\alpha+2)^{2}}+\frac{1}{(\alpha+1)^{2}}\left[1-n\left(\frac{\alpha}{\alpha+1}\right)^{2} \frac{1+\theta(\alpha+1)}{1+\theta \alpha}\right]\right\} .
$$

Moreover, using (3.1), we can determine all raw and central moments of $X$. In addition, measures such as skewness and kurtosis can also be derived. However, the mathematical expressions become intractable.

\section{Parameter Estimation}

\subsection{Method of Moments}

For a random sample $X_{1}, \ldots, X_{N}$ of size $N$ from the log-Lindley binomial distribution, let $m_{1}$ and $m_{2}$ be respectively the first and second raw sample moments. That is, let $m_{1}=\frac{1}{N} \sum_{i=1}^{N} X_{i}$ and $m_{2}=\frac{1}{N} \sum_{i=1}^{N} X_{i}^{2}$. Let further $u_{1}$ and $u_{2}$ be the corresponding factorial 
sample moments defined as $u_{1}=m_{1}$ and $u_{2}=m_{2}-m_{1}$. By setting the first two factorial moments equal to $u_{1}$ and $u_{2}$ we have

$$
n\left(\frac{\alpha}{\alpha+1}\right)^{2} \frac{1+\theta(\alpha+1)}{1+\vartheta \alpha}=u_{1}
$$

and

$$
n(n-1)\left(\frac{\alpha}{\alpha+1}\right)^{2} \frac{1+\theta(\alpha+2)}{1+\vartheta \alpha}=u_{2}
$$

Upon solving (4.1) and (4.2) for $\alpha$ and $\theta$, after some algebra we find that $\hat{\alpha}_{M}$, the moment estimator of $\alpha$, is the positive root of the quadratic equation

$$
\left(2 u_{1}^{\prime}-u_{2}^{\prime}\right) \alpha^{2}+4\left(u_{1}^{\prime}-u_{2}^{\prime}\right) \alpha+2\left(u_{1}^{\prime}-2 u_{2}^{\prime}\right)=0,
$$

and the moment estimator of $\theta$ is given by

$$
\hat{\theta}_{M}=\frac{u_{1}{ }^{\prime}\left(\hat{\alpha}_{M}+1\right)^{2}-\hat{\alpha}_{M}^{2}}{\hat{\alpha}_{M}\left(\hat{\alpha}_{M}+1\right)\left[\hat{\alpha}_{M}-\left(\hat{\alpha}_{M}+1\right) u_{1}{ }^{\prime}\right]^{\prime}},
$$

where $u_{1}^{\prime}$ and $u_{2}{ }^{\prime}$ are defined as $u_{1}{ }^{\prime}=\frac{u_{1}}{n}$ and $u_{2}{ }^{\prime}=\frac{u_{2}}{n(n-1)}$.

\subsection{Maximum Likelihood Estimation}

Let $f_{x} ; x=0,1, \ldots, n$ be the observed frequencies. Then the log-likelihood can be expressed as

$$
\ln L=\sum_{x=0}^{n} f_{x} \ln [P(x)]
$$

Define $S_{x} ; x=0,1, \ldots, n$ by

$$
S_{x}=\frac{P(x)}{\left(\begin{array}{l}
x \\
n
\end{array}\right)}
$$

then from (4.3),

$$
\ln L=\sum_{x=0}^{n} f_{x} \ln \left[s_{x}\right]+\text { constant. }
$$

Equation (4.4) must be maximized in order to find the maximum likelihood estimates of the parameters. Unfortunately, differentiating (4.4) with respect to the two parameters and and setting the derivative equal to 0 leads to a set of intractable equations. However, there are many standard programs that can be used for direct optimization of (4.4) or solving the likelihood equations. For example, the function mle in R provides a direct optimization of the likelihood function using Nelder-Mead quasi-Newton and conjugate gradient algorithm. See Nocedal and Wright (1999) for more details. 


\section{Application in Developmental Toxicology}

Developmental toxicology is the branch of science that is the study of adverse developmental effects of drugs, chemicals, diet, and environmental agents (Mattison, et al., 2003). Bioassay experiments are designed using laboratory animals, usually rats and mice, to assess the effe3ct of maternal exposure to the offspring. The general design of developmental toxicity experiments consists of using between 20 to 25 pregnant female rodents. The animals are randomly assigned and exposed to up to four dose levels of the chemical agent, including the placebo controls. The exposure occurs during a critical time of the gestation period. To examine the physical effects of the chemical agent, the animals are either observed through lactation or sacrificed just before term and the uterine content is examined for a variety of effects including death/resorption, fetal weight, and malformation. A fetus that is not dead or resorbed is called viable. One of the main outcomes of interest is the number of viable fetuses with at least one malformation at each dose group. Generally, this number increases with increasing dose. More importantly, however, it has been observed that fetuses from the same litter behave more similar than fetuses from different litters. Ignoring this so-called intralitter correlation can grossly affect the assessment of risk. Several statistical models have been proposed for the analysis of data from developmental toxicity studies and for a description of these models we refer to Razzaghi (2020). Perhaps the earliest attempt to model dichotomous outcomes from developmental toxicity studies is by Williams (1975). Assume that the experiment consists of $g$ dose levels plus the control

$$
0=d_{0}<d_{1}<\ldots<d_{g} .
$$

Suppose that $m_{i} ; i=0,1, \ldots, g$ pregnant female animals are exposed to the dose $d_{i}$ of a developmental toxicity agent during gestation. Let $n_{i j}$ and $X_{i j}$ be respectively the litter size and the number of affected fetuses of the $j$ th mother in the $i$ th dose group. Then if we denote by $\pi_{i j}$ the probability of an adverse effect e.g. malformation, then the conditional distribution of $X_{i j}$ given $\pi_{i j}$ is binomial. Assuming a beta distribution for $\pi_{i j}$, the unconditional distribution of $X_{i j}$ becomes beta-binomial which was utilized by Williams (1975) and others. See also Chen and Kodell (1989). Now, if instead of the beta distribution, alternatively we assume that the distribution of the response probabilities $\pi_{i j}$ follow a log-Lindley distribution, then the unconditional distribution of the number of responses in the $j$ th litter of the $i$ th dose group is log-Lindley binomial. Specifically, if we assume that the distribution of the probability of response in the $i$ th dose group is

$$
f\left(\pi_{i}\right)=\frac{i^{2}}{1+\theta_{i} \alpha_{i}}\left(\theta_{i}-\ln \left(\pi_{i}\right)\right) \pi_{i j}^{\alpha_{i}-1}, 0 \leq \pi_{i} \leq 1, \alpha_{i}>0, \theta_{i} \geq 0 ; i=0, \ldots, g,
$$


then the unconditional distribution of $X_{i j}$ is given by

$$
\begin{aligned}
P\left(X_{i j}=x_{i j}\right)= & \left(\begin{array}{l}
x_{i j} \\
n_{i j}
\end{array}\right) \frac{i^{2}}{1+\theta_{i} \alpha_{i}} \sum_{l=0}^{n_{i j}-x_{i j}}(-1)^{l}\left(\begin{array}{c}
l \\
n_{i j}-x_{i j}
\end{array}\right) \frac{1+\theta_{i}\left(\alpha_{i}+x_{i j}+l\right)}{\left(\alpha_{i}+x_{i j}+l\right)^{2}} ; \\
& x_{i j}=0,1, \ldots, n_{i j}, \quad j=1, \ldots, m_{i}, \quad i=0, \ldots, g .
\end{aligned}
$$

\section{Examples}

In this section we illustrate the application of the log-Lindley binomial distribution using a real experimental data. For the ease of comparison, we use the same data set that was utilized by Williams (1975) for illustration of the application of the beta-binomial distribution. The data set is from a case-control teratological experiment described in Weil (1970). Pregnant female rats were divided in two groups fed a control diet or exposed to a dose of a teratogen through gestation and lactation. Each group consisted of 16 rats and for each litter the numbers $n$ of live pups after 4 days and $x$ after 21 days of lactation were recorded. Table 1, reproduced from Williams (1975) displays the data. For simplicity and purpose of illustration, we assume that $\theta=0$. That is, we fit the special case of the log-Lindley binomial distribution that was considered in Grassia (1977). Using the mle function in R with the Nelder-Mead method of optimization, we found that for the control animals, the estimate of $\alpha$ is given by alpha ${ }_{C}=18.025$ with a standard error of 5.5636 and a log-likelihood of -21.390 . For the treated animals, we have $\hat{\alpha}_{T}=6.175$ with a standard error of 1.589 and log-likelihood of -34.569 . Figures 2 and 3 depict the likelihood profiles for the control and treated data respectively. The values of the Akaike Information criteria (AIC) were 44.780 and 71.138 for control and treated groups. In contrast to Williams (1975), the estimated parameters result in a J-shaped distribution for the control group and a bell-shaped distribution for the treated group. Figure 4 displays the graphs of the fitted distributions for the two groups. To test for the treatment effect i.e. testing the hypothesis that $a l \hat{p h} a_{C}=a l \hat{p h} a_{T}$, similar to Williams (1975), using the combined data, a single log-Lindley binomial distribution was fitted to the 32 observations. It was found the joint estimate of $\alpha$ is given by alpha $a_{C}=a l \hat{p h} a_{T}=9.775 \pm 1.900$ with a log-likelihood of 59.623 and AIC value of 121.245. Figure 5 shows the likelihood profile of the combined fit. We thus have

$$
2\left(L_{1}-L_{0}\right)=2(-21.390-34.569+59.623)=7.329,
$$

which has a chi-square distribution with one degree of freedom. This leads to a highly significant treatment effect $(p=0.0068)$. 
Table 1: Number of live pups and litter sizes.

\begin{tabular}{rrrrrrrrrrrrrrrrrr}
\hline \multirow{2}{*}{ Control } & $\mathrm{x}$ & 13 & 12 & 9 & 9 & 8 & 8 & 12 & 11 & 9 & 9 & 8 & 11 & 4 & 5 & 7 & 7 \\
& $\mathrm{n}$ & 13 & 12 & 9 & 9 & 8 & 8 & 13 & 12 & 10 & 10 & 9 & 13 & 5 & 7 & 10 & 10 \\
& $\mathrm{x}$ & 12 & 11 & 10 & 9 & 10 & 9 & 9 & 8 & 8 & 4 & 7 & 4 & 5 & 3 & 3 & 0 \\
Treated & $\mathrm{n}$ & 12 & 11 & 10 & 9 & 11 & 10 & 10 & 9 & 9 & 5 & 9 & 7 & 10 & 6 & 10 & 7 \\
\hline
\end{tabular}

Source: Williams (1975)

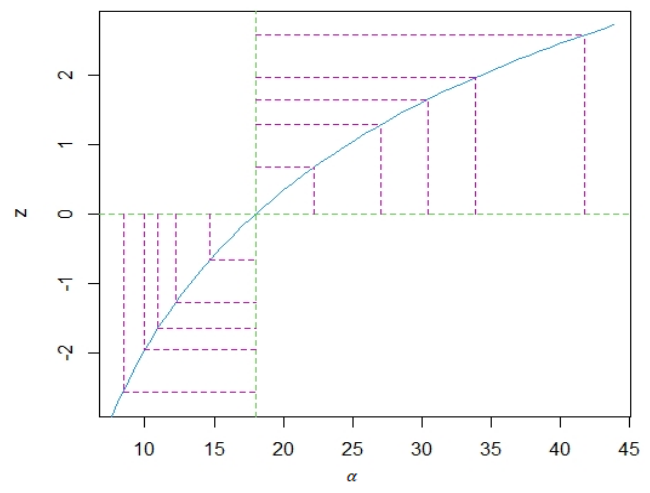

Figure 2: Likelihood Profile for Control Data.

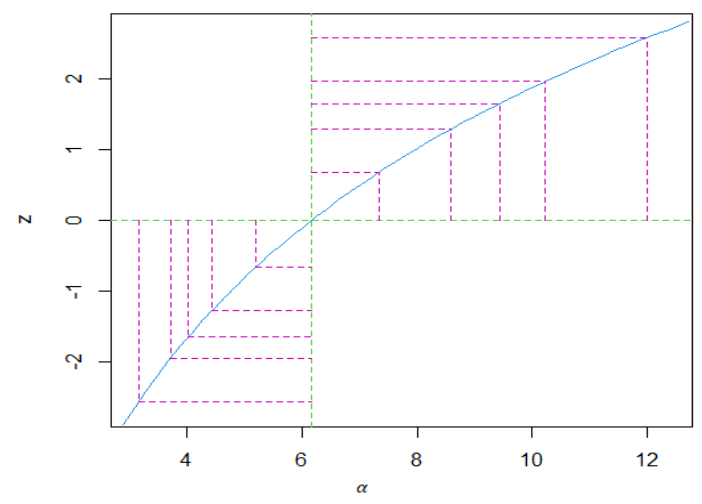

Figure 3: Likelihood Profile for Treated Data. 


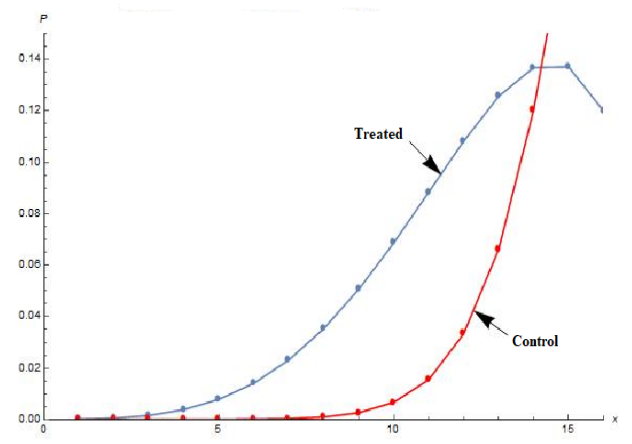

Figure 4: Fitted Distributions for Control and Treated.

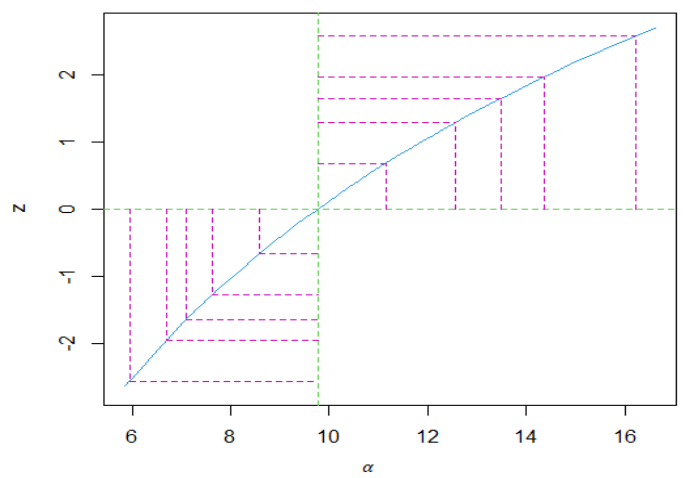

Figure 5: Likelihood Profile for the Combined Data.

\section{Conclusion}

When the outcome of an experiment is quantal and the data are observed in clusters, the traditional approach for modeling is to apply the beta-binomial distribution. This approach has found widespread popularity in many different applications due to the flexibility and interesting properties of the beta-binomial model. In this paper, we introduce an alternative approach for modeling clustered binary outcomes. Using a log-Lindley distribution instead of the traditional beta as the mixing distribution, we find a new log-Lindley binomial model. This alternative is flexible for modeling and 
has many interesting properties. Specifically, it was demonstrated that the model can successfully be applied for modeling experimental data from developmental toxicity experiments. Although the beta-binomial model has extensively been applied for risk assessment, it remains to be seen how the new proposed log-Lindley binomial model can be utilized in a similar application.

The purpose of this paper is not so much in introducing a competitor for the betabinomial distribution. The aim is not to show whether or not the log-Lindley binomial distribution works better or worse in practice. Rather, the goal is to bring the new alternative to the attention of the statistical community.

One drawback of the beta-binomial distribution is that the intra-cluster correlation is inherently positive and although it can successfully be applied for modelling overdispersed data, the distribution has limited applicability when the data exhibit underdispersion. For this reason, several extensions of the beta-binomial model have been introduced to make it amenable to modeling underdisperesed data. These areas together with other properties of the log-Lindley binomial distribution can be some topics of investigation for future.

\section{References}

Berson, E. L., Rosner, B., and Simonoff, E. (1980), An outpatient population of retinitis pigmentosa and their normal relatives: risk factors for genetic typing and detection derived from their ocular examination. American Journal of Ophthalmology, 89, 763-775.

Chen, J. J., and Kodell, R. L. (1989), Quantitative risk assessment for teratological effects. Journal of the American Statistical Association, 84, 966-971.

Gomez-Deniz, E., Sordo, M. A., and Calderin-Ojeda, E. (2014), The log-Lindley distribution as an alternative to the beta regression model with applications in insurance. Insurance: Mathematics and Economics, 54, 49-57.

Grassia, A. (1977). On a family of distributions with argument between 0 and 1 obtained by transformation of the gamma and derived compound distributions. Australian Journal of Statistics, 19, 108-114.

Jorda, P., and Jimenez-Gamero, M. D. (2016). A note on the log-Lindley distribution. Insurance: Mathematics and Economics, 71, 189-194. 
Lindley, D. V. (1958). Fiducial distribution and Bayes' theorem.Journal of the Royal Statristical Society, Series B, 20, 102-107.

Lindley, D. V. (1965). Introduction to Probability and Statistics from a Bayesian Viewpoint, Part II: Inference. Cambridge University Press, New York.

Nocedal, J., and Wright, S. J (1999). Numerical Optimization. Springer, New York. R Core Team (2012). R: A language and environment for statistical computing. R Foundation for Statistical Computing, Vienna, Austria. ISBN 3-900051-07-0, URL http://www.Rproject.org/

Razzaghi, M. (2020). Statistical Models in Toxicology. CRC Press, Boca Raton, FL.

Rosner, B. (1982). Statistical methods in ophthalmology: an adjustment for the intraclass correlation between eyes. Biometrics, 38, 105-114.

Smith, D. M. (1983). Maximum likelihood estimation of the parameters of the betabinomial distribution. Applied Statistics, 32, 192-204.

Statistical Analysis System SAS (2014). Version 9.4. Cary, NC: SAS Institute Inc.

Wilcox, R. E. (1981), A review of the beta-binomial model and its extensions. Journal of Educational Statistics, 6, 3-32.

Williams, D. A. (1975), The analysis of binary responses from teratological experiments involving reproduction and teratogenicity. Biometrics, 31, 949-952.

Zakerzadeh, H., and Dolati, A. (2009), Generalized Lindley distribution. Journal of mathematical Extension, 3, 13-25. 